Tribe, R. \& Thompson, K. (2009) Exploring the Three Way Relationship in Therapeutic Work with Interpreters. International Journal of Migration, Health and Social Care, 5,2, 13-21

"The answer to many of these difficulties is of course to draw upon the interpreters' knowledge and experience as a resource. Don't be intimidated by it. Use it. But do not use it uncritically or unquestioningly. Having admitted that the interpreter is a source of expertise it is easy for the therapist to allow the interpreter to take over and in some sense 'lead' the therapeutic process. It is therefore important to remember that the interpreter is not a counsellor or psychotherapist" Blackwell (2005:86)

Exploring the Three Way Relationship in Therapeutic Work with Interpreters.

Abstract

In a companion paper, the authors have argued that therapeutic work with interpreters has been viewed more negatively than is warranted, and that the inherent advantages of this way of engaging with the Non English speaking client have been minimized or ignored (Tribe and Thompson, submitted). This paper seeks to explore the aspects that may underlie the reluctance of clinicians to engage with therapeutic work with interpreters. Difficulties often appear to be centred on the anxieties provoked by working in the three way therapeutic relationship rather than in the traditional therapeutic dyad. It is also possible that the highly traumatized nature of some clients, who may be refugees or asylum seekers fleeing from political violence, also complicates 
such work. The intention in this paper is to consider both the dynamics of the three-way relationship and the impact of traumatic experience, when relevant, on therapeutic work with interpreters and suggest how the pulls inherent in such work may be managed. Conclusion: It is hoped that by exploring these problematic areas, some light can be shed on the difficulties that all clinicians can experience but can equally overcome.

\section{THE POWER OF ALLIANCES}

One of the commonalities across therapeutic approaches is the emphasis on the centrality of the relationship between therapist and client, something that unites practitioners from all the great traditions of counselling and psychotherapy. In traditional psychotherapy, the key relationship is dyadic, but the dyad increases to a triad once an interpreter is introduced. It is widely agreed that the most helpful approach to therapeutic work with an interpreter is to view this as a three way relationship (rather than representing the interpreter as a kind of human translating machine neutrally placed in a traditional relationship between therapist and client) (Baker \& Briggs, 1975; Westermeyer 1990; Haenal 1997). Baker \& Briggs (1975) and many authors following them have viewed this as a triangular relationship, and have represented it as ideally an equilateral triangle in which the distance between each of the three participants is essentially the same. Difficulties arising in the relationship have often been presented as the result of changes to the equal distance between the participants. As Haenal (1997:68) notes: "We soon found that there were patient/therapist/interpreter triads that were stable and 
conducive to the progress of therapy, while there were others that were not, in which it was sometimes uncertain whether the cause of stagnation was to be sought in the patient, the therapist, the interpreter or in all three". In our view, this represents a rather rigid view of the ideal dynamics in therapeutic work with interpreters. In fact, the distance between the three participants is always in motion, shifting depending on the material being addressed, the stage of therapy involved, the dynamics of the individuals and so forth. As

noted by Spector, Briedis, \& Rebori (unpublished) there is an ongoing process of splitting and pairing when working with an interpreter, and these pairings may change at different points in the therapy work. For example, a number of potential pulls can lead to greater closeness between the interpreter and the client or less commonly, between clinician and interpreter. The least common potential alliance appears to be that between clinician and client but some examples of this are also listed:

\section{Client and Interpreter Alliances:}

1. History, culture, politics and similarity of experience between client and interpreter can give rise to greater closeness, especially early on in therapy. In many cases, writers have seen this as a helpful aspect in establishing the therapeutic alliance (Raval, 1996; Saxthorp \& Christiansen, 1991) It can be enhanced by the habit of some clients of addressing interpreters (or even relating to interpreters) as if they were members of the family, calling them uncle or aunty, big sister etc. Interestingly a study in London (Alexander, Edwards, Temple, Kanani, 
Zhuang, Miah \& Sam, 2004) showed that many health service clients much preferred the idea of a family member acting as interpreter and it may be that this habit of referring to the interpreter in familial terms is linked to this preference. This implied relationship can appear to give the interpreter power in the relationship with the client, but in fact it is often experienced by the interpreter as burdensome closeness or a pressure to become involved in client problems and "rescue" them (see below) $)^{\mathrm{i}}$.

2. As a result of closeness between interpreter and client, many writers note that practitioners, particularly those new to working with interpreters, may end up feeling "left out". Dearnley (2000: 21) describes occasions when she has felt herself a "gooseberry...feeling that the client and interpreter would be happier to lose me whilst they could reminisce about the country and culture they have both lost".

3. In some cases the client may not understand or appreciate the practitioner's way of working and may become closer to the interpreter and confide their doubts about the approach as a result. This can be difficult to manage for the interpreter. However, Miller, Martell, Pazdinek, Carruth, \& Lopez (2005) also report a case in which the interpreter thought a practitioner's use of a grounding technique was "stupid" and told this to the client. Clearly there is potential to initiate an alliance against the "stupid doctor" from either client or interpreter. 
4. The authors have both experienced times when clients have chosen to confide a "secret" in an interpreter that they claim (at least initially) they do not want to be disclosed to the clinician. In some cases, it arises when a client wants to reveal information that they think may be too traumatic, shameful, or culturally complex to express to a clinician directly. In other cases, the "secret" may have political implications that the client feels it is dangerous to share with the clinician as a representative of the host country (for example, in relation to asylum claims or specifics of nationality).

\section{Clinician and Interpreter Alliances:}

1. In some cases, there can appear to be commonality between the clinician and interpreter, particularly if the latter has lived some time in the second country and taken on a professional identity. In one case, Thompson recalls that she worked with an interpreter who told her confidentially after the session that the client they had just assessed was a "bogus asylum seeker" for whom he had already interpreted at the hospital. The interpreter felt that it was important that she be informed about this although he had no specific evidence to support his ideas. Further discussion revealed his need to distance himself from the client, who he felt had been racist towards him and for whom he had little empathy (see below for further on this example in the light of wider power dynamics). 
2. Haenal (1997) describes a situation in which an interpreter with therapy needs of her own became very admiring of the clinician with whom she was working, telling him her life story in instalments and bringing gifts while simultaneously depreciating the client.

3. When working with interpreters, the authors have also noted that it is important to track the way in which the therapist's engagement with the client may be mirrored by the interpreter. For example, when the clinician is feeling bored or frustrated in the work, can this be detected in the stance of the interpreter towards the client? In many cases, we have found that such processes are reflective of an underlying alliance between clinician and interpreter which is not necessarily unmanageable, but requires monitoring.

\section{Clinician and Client Alliances:}

1. In general this sort of alliance is less likely to occur given the fact that the two parties have less direct contact and often do not share sufficient language in common. That said, there have certainly been occasions when the authors have noted a tendency to "blame" the interpreter, and sometimes both client and clinician can take refuge in this together. Thompson recalls one example when a client, who had

worked for a number of sessions with the same interpreter, became angry with the clinician. The day following the session, a relative called and explained that he wished to change the interpreter as he felt she 
was not good enough to translate him fluently. In this case, the fact that the clinician knew the interpreter well and could track the source of the disturbance with clarity, meant that the difficulty could be considered in the light of their three way relationship. It is to be imagined, however, that in another situation burying anger with the clinician by deciding the interpreter is the problem might lead to a dismissal of the interpreter.

2. In other cases, there may be reasons why the client seeks a primary alliance with the clinician (perhaps to the exclusion of the interpreter). They may see themselves as a professional, sharing an educated background with the clinician, which they may believe the interpreter does not have. Tribe (1999) reports the case of Mr Lopez, who had recently arrived in Britain and spoke little English. She describes beginning to see him with an interpreter with whom she had worked on many occasions and in whom she had great trust and respect. From early on in his sessions, her client quickly began to try and avoid using the interpreter by explaining things direct to her in broken English, or in his own language but very slowly and expressively, and finally by drawing on pieces of paper to try and get his message across. She states that she was highly aware that her client hardly looked at the interpreter and appeared to be trying to largely ignore his presence. On further exploration it emerged that Mr Lopez felt that the interpreter could not understand him, as he believed he came from a "poor 
uneducated place" while himself and his clinician were similar in educational background.

\section{UNDERSTANDING THREE-WAY DYNAMICS}

When writing about the dynamics that arise in therapeutic work with interpreters, many writers have made reference to the psychodynamic concepts of "transference" and "counter-transference", often seeking to track the alliances formed as if they were expressions of past familial relationships. In the example of the admiring interpreter (point 2, section on clinicianinterpreter alliances) the author explains the interpreter's behaviour as a reflection of a paternal transference to the clinician (Haenal, 1997). We would argue that the situation is often more complex than this and tends to reflect the way in which power relations are working within the three way relationship itself, but also in the wider world. In this we would agree with Miller et al (2005) who report that they eventually discarded the terms "transference" and "countertransference" in favour of the phrase "complex emotional reactions".

In the experience of these authors, clients often respond to their interpreters in ways which appear more reflective of recent experiences of ethnic conflict between groups or the ways in which power is negotiated at a societal level rather than early interpersonal experiences. It is therefore important to view what is taking place within therapy with a wide lens, and explore which structures of power may be being reflected within the dynamics of the three way relationship. While these may include mirroring of family dynamics, the 
wider structures of power within society are also of relevance. The positioning of gender or age within the power hierarchies may be important to consider, as the marginalization of ethnic minority groups, and discrimination against asylum seekers and refugees, all of which can be key influences on the dynamics of the therapeutic triad.

In the example given above, of the interpreter who informed Thompson that her client was a "bogus asylum seeker", the interpreter was an older man of Asian origin working in his second and third languages (a middle Eastern language and English) and had been treated somewhat disdainfully by the client in a way that reflected forms of discrimination in the society the client had fled. Thompson was interested by this, and wondered about the timing of his disclosure, which broke confidentiality about his past work with the client, and what it might represent in terms of his feelings of alliance or identification with her against the client. In fact, work with this client and interpreter was not successful and the client failed to attend appointments after one further session in which he stated that he felt Thompson did not believe in his story of past violence, something clearly reflecting the issue raised by the interpreter. In this case, it proved almost impossible for clinician and interpreter to explore the problematic dynamics in the therapy situation, and the authors would argue that this led to the collapse of any healthy alliance, and the end of therapeutic work. This example demonstrates the way in which issues of power and disempowerment can arise in relation to the political contexts of both the society left (first country) and the society of migration or exile, and 
the need to consider such issues in therapeutic work from the perspective of the three parties involved.

Power relations in the triadic relationship between shared clinician, interpreter and client are ambiguous. Westermeyer (1990:747) describes this by saying: "The three roles of clinician, interpreter, and patient are not the same in terms of professional, legal or symbolic status. Although the patient can "hire or fire" the other two, there exists the imposing physical fact of two staff against one patient. The easily threatened clinician, faced by two persons of the same language and usually the same culture or race, may feel overwhelmed. The clinician is legally and ethically in charge of the interpreter and the interview process, a fact that must be appreciated by the interpreter". As Raval (1996) notes, the interpreter is in a strange position hierarchically, sharing in some sense the skills and position of the clinician (skills in listening, availability, professional status, albeit at a lower level than the clinician) but without the authorization to take the lead in any work undertaken.

Similarly, Patel (2003) notes that while interpreters may appear to have power, in that they are crucial for communication between client and interpreter, they in fact lack personal efficacy, as they cannot speak with their own voice and can initiate only as far as they are permitted by the other two parties, particularly the clinician. She clarifies this stating: "In fact the therapist remains the main driver with the interpreter as co-driver who is sometimes allowed to drive, using their own expertise and knowledge" (Patel 2003:228) 
While this is certainly the case, it is interesting to reflect on some examples from the literature which indicate the way in which the interpreter's stance and mode of translating makes use of the communicative power that they do have. A number of writers have noted the way in which interpreters may soften the emotional impact of the difficulties of clients for whom they are interpreting (Sabin, 1975). Westermeyer (1990) remarks that "it frequently occurs that fellow expatriates attempt to "normalize" the patient's psychopathology by not translating material relevant to suicidal, paranoid ideation or other topics that embarrass the untrained translator or cause the translator to "protect" the patient from "medical authorities" (1990:748).

Similarly, Farooq \& Fear (2003) remark on the way that interpreters may "translate" symptoms that they see as culturally located and difficult to fit within Western diagnostic frames, so that they are easier for clinicians to understand and make use of. In this regard, it is also important for clinicians to "be aware of what they represent for their client" (Holder 2002) and also for their interpreter in terms of the power structures inherent in society. Thus working cross culturally, one needs to think about the way in which the racial and cultural biases of the past continue to impact on the ways in which services are set up and delivered and the way in which clients may believe they need to engage with services (Rechtman 2000). "Softening" clients for clinicians and clinicians for clients might then be seen as a way of negotiating power differentials reflecting the position of the interpreter as a bridge between two cultures, and two power positions, attempting to manage 
sometimes disparate worldviews and identities in a relationship in which their role is to "interpret" and their pay depends on making a coherent job of this.

Given the difficulties in managing the pull of power relationships and the pairings that result, the authors would argue that any of the two-way alliances above spells trouble for good three way work, as does the tendency to view the interpreter as an 'interpreting machine' or a form of translation software (Tribe 1999) sidelining their presence and personhood in an attempt to create something more like a dyadic relationship. Miller et al (2005) point out that this is enhanced by asking interpreters to use the first person rather than third person in interpreting, requiring them to be as unobtrusive as possible by placing them out of view and never allowing them to speak or offer any personal input to the therapeutic process. This stance towards an interpreter views them as "an unfortunate necessity, a potential obstacle to genuine therapeutic contact with the client' (2005:30) rather than embracing the contribution that an interpreter can make to the therapeutic process.

\section{THE IMPACT OF TRAUMATIC MATERIAL ON THE THREE WAY RELATIONSHIP}

When thinking through the dynamics and complexities of the three-way therapeutic relationship involving an interpreter, it is also important to keep in mind the way in which traumatic material arising in therapy can impact upon the dynamics. As an increasing number of non English speaking clients in the UK are asylum seekers or refugees, often referred for psychological therapy 
for difficulties arising from past traumatic experiences, it becomes crucial to consider the influence of this on the three way relationship. It has also been argued that the lack of training for interpreters undertaking work with traumatized clients can place them at substantially greater risk of vicarious traumatisation (Becker \& Bowles 2001).

The political context in which a refugee or survivor of violence finds themselves can also impact on therapeutic work. Haenal (1997) describes the problems that can arise when an interpreter's sympathies restrict the available ways of being open to a client. In his example "the patient was validated in his militant role by the interpreter, which prevented him from consciously re experiencing the personal and narcissistic injury that he had experienced under torture" (1997:69). More commonly, it may be the interpreter who is left feeling he or she has not fulfilled a political duty in having left the shared country and lived safety elsewhere, and there may be a sense of obligation to the client out of conscious or unconscious feelings of solidarity.

Questions of power are of acute relevance when working with those who have experienced persecution, who may have been the absolute victims of the violence, humiliation and intimidation of others. Such experiences leave their mark, and are often echoed by the marginalization and discrimination faced as an asylum seeker attempting to relocate to a safer society. Powerlessness is also a feature of the situation in which one is silenced by the need to use an interpreter (Patel, 2003) and this combination of factors can lead many clients, 
particularly refugee clients, to present themselves as victimized, without resources and entirely passive in the face of difficulties.

Although this power dynamic can be seen as inherent to the systems clients have fled and the situation they face in this country, clinicians and interpreters are in a position to challenge or collude with this sense of the client as passive and victimized (Blackwell, 2005). It is also crucial to manage the pull towards the roles that can be adopted following traumatic experience, with a tendency for victimization to repeat itself when those involved are drawn towards a stance of victim, persecutor or, perhaps most difficult to manage, the pull to rescue the client from their difficulties (Blackwell, 2005). In a triadic relationship there tends to be less flexibility for the way in which these three roles can change so that rather than therapist and client moving between these positions, they can become fixed. Both authors have had experience of interpreters becoming drawn into the rescuer role, and then reproaching the clinician for not doing enough to help the client with practical difficulties.

A further example can be used to illustrate this. On one occasion, a colleague worked therapeutically with a young woman who had survived torture in Turkey. At a certain point in the work, there was an angry interchange between the client and her female interpreter following which the client refused to have further sessions with the interpreter and therapeutic work ceased, despite her very positive feelings about the therapist. This was viewed as an isolated incident and it was felt that the interpreter had behaved inappropriately, and that the client had been the victim of a difficult situation 
(Ballance, 2007, personal communication). A later referral to another service led to the client being assessed by Kate Thompson and seen for therapeutic work by a Turkish speaking colleague. Despite reporting very positive feelings about the assessment process, the client quickly stopped her sessions with her counsellor and sought re-referral to work with an English therapist. It appeared that there was a wider difficulty that seemed to reflect the client's sense that only a non-Turkish person was to be trusted. This had initially expressed itself by a rejection of the person of the interpreter but now revealed itself as a more widespread belief about the safety of relationships with individuals from the country she had fled. Thus, her experiences of trauma and violence, inherently political in nature, continued to influence her experiences in a new country, detracting from her efforts to seek help and polarising her between views of helpers as either potential persecutors or rescuers.

\section{BUILDING AN EFFECTIVE THREE WAY RELATIONSHIP}

The authors hope that the examples above have illustrated the difficulties that can arise when the dynamics are not managed well in a three way relationship (particularly with the addition of issues of trauma and powerlessness). This should come as no surprise to those who are involved in work with families, with couples or who do joint work with other professionals, where an emphasis on containing processes of "splitting" forms one of the key concerns of the work. It is interesting that this focus on avoiding splitting does not seem to have been invoked when considering work 
with interpreters. It may be that the tendency of clinicians to underestimate the importance of the interpreter to the triadic relationship is responsible for this oversight, and that interpreters have lacked sufficient training to raise these issues with clinicians themselves.

In our view, which echoes that of many of the writers quoted in this paper, the interpreter is an integral part of a three way alliance: "the interpreter is an important witness to the client's experience, and the gradual unfolding of the client's story reflects a growing sense of trust not only between client and therapist but also between client and interpreter" (Miller et al, 2005:30). What is crucial in this work, however, is building a healthy three way relationship. Much as might be the case when working therapeutically with a couple, where the struggle is to maintain a three way relationship (or in conjoint therapy with couples, a four way relationship) without splitting into alliance with either party (Ruszczynski 1993), the same challenge obtains in working with interpreters.

This raises an interesting possibility that the authors would like to consider further. Much of the literature argues for the work to be visualized as an equilateral triangle, with equal closeness between each of the three parties and no pulls towards specific excluding alliances. However, taking therapeutic work with couples as a comparative case, it has been argued that the best way to think about work with a couple, is to see it as a relationship between therapist and couple rather than a three way relationship with two separate persons (Ruszczynski 1993). This can aid in containing the splits 
that might otherwise arise, helping the clinician spot ways in which tensions might be driving an alliance with one or other member of the couple.

Clearly the situation is different when working with an interpreter and client, but there is something interesting about the idea of building a fixed alliance in advance, in the service of therapy, to check the pull towards more unhelpful and excluding patterns. It may, then, be more beneficial to develop situations in which the clinician and interpreter are slightly closer to one another than they are to the client, and can share their observations of the work and support one another, while reflecting on any pulls to unite themselves unhelpfully with the client. This may prove to be a more constructive model to employ than one of absolute equality of distance, which would seem more prone to the development of unhelpful alliances.

As stated above, there are some difficulties in invoking concepts of transference and counter-transference in considering the patterns that may arise in such work. In some cases, there may appear to be elements of family patterns involved which help locate the therapeutic relationship in the familiar but in others, the three-way pattern appears to reflect more global structures of power used to counteract the effects of victimization or hold both resilient and vulnerable sides of a client in tension through ongoing work. Some examples are given to illustrate this:

1. Thompson recalls working with an older male interpreter and a young male client both from Ethiopia. The interpreter was 
sometimes a little unclear in his interpreting in English, and she became concerned that he might not be getting her message across very well. As a result, she challenged the interpreter rather sharply in one session and he was put in a position of defending his interpreting in front of the client. It became clear that this had been unsettling for the client. Rather than seeming to him like the therapist was concerned to ensure they could communicate, there was a sense in which he had seen two individuals on whom he was seeking to rely for safety arguing with each other. Thompson had been aware that this rather shy young man seemed to appreciate the way in which she and the interpreter formed a coherent couple (rather like a "Mum" and "Dad") and the sight of her contradicting the interpreter (who was a respected older man in the community) may have been very uncomfortable, undermining the need for the two professionals to operate as a "containing pair".

2. In work with women survivors of sexual violence, both authors have experienced the impact of working as a female clinician with a female interpreter. The three way relationship becomes a collective of women, with some commonality of experience, allowing the witnessing of traumatic experience to work with much greater effect. Bot \& Wadensjo (2004:375) similarly state that they have heard patients say that "they liked the fact that two people heard their stories. The more people that know about these terrible things the better", again suggesting that the potential for witnessing can be 
greater when working with interpreter. It also add the safeguard for many clients of feeling confident that their story is likely to be believed by clinicians whose own background may not incline them to know the level of violence or human rights abuse present in a given situation.

3. Ballance reports the case of a young man with whom she worked with an older male interpreter. As the therapeutic relationship developed, it was clear that her client had bonded in different ways to her and to the interpreter. He seemed to relate to his therapist as a maternal figure with whom he could experience feelings of vulnerability but his stance towards the interpreter reflected a view of the latter as worldly and humorous, an avuncular figure with whom he could enjoy jokes and show a more cynical and perhaps resilient side of himself. Ballance makes the point that such a "cotherapist" role for an interpreter is only possible with a very experienced interpreter whose personal skills allow them to take on part of the therapeutic role and where joint thinking between clinician and interpreter is possible (Ballance, 2003).

Spector et al (unpublished) suggest that joint supervision for the interpreter and clinician together may help in managing splitting and related issues. This would certainly be an interesting way to manage the relational pulls we have described although it may be that individuals require separate supervision in keeping with their different roles and that a joint process meeting is more 
likely to meet the needs of all parties. The fact that interpreters are often employed sessionally, that organisations rarely recognise their central importance and are unlikely to pay for their attendance in such meetings makes it unlikely that such a model will however be realised. That said, a stance which places the interpreter as a vital component of the therapeutic relationship and as a colleague intent on managing splits of this kind alongside the clinician is much more likely to contain such alliances. Such an approach takes for granted the experience and willingness of both clinician and interpreters to address these issues.

\section{CONCLUSION}

Overall, then, this paper has argued that the aim of therapy with an interpreter is to offer a relationship with a co-working couple, therapist and interpreter, who can work effectively and thoughtfully to manage the changing shape of the triangular relationship between the three parties. The closeness of clinician and therapist, and their space to reflect jointly, are the elements that can be used to manage and negotiate any pulls in the three way relationship

Given the complexities that can arise in three way therapeutic relationships with interpreters, it is perhaps not surprising that many clinicians have seen the task of therapy through interpreter as difficult and challenging. The authors hope that this paper has illustrated the types of potentially difficult dynamics that can arise but has also offered useful pointers as to how these difficulties might be circumvented. A companion paper explores the ways in 
which working with an interpreter can add value to therapeutic work and makes recommendations for enhancing the effectiveness of this work (Tribe and Thompson, submitted).

\section{REFERENCES:}

Alexander, C., Edwards, R., Temple, B., Kanani , U., Zhuang, L., Miah, M. \& Sam, A. (2004). Access to services with interpreters, user views. York: Joseph Rowntree Foundation

Baker, R. \& Briggs, J. (1975). Working with interpreters in social work practice. Australian Social Work 28 (4), 31-37.

Ballance, G. (2003). Paper presented at Seeking Asylum, a day workshop at the Society for Analytical Psychology. London, 18 October 2003.

Becker, R \& Bowles, R (2001). Interpreter's experience of working in a triadic psychotherapy relationship with survivors of torture and trauma: Some thoughts on the impact on psychotherapy. In B. Raphael \& A. Malak (Eds), Current Issues in Transcultural Mental Health Diversity: Mental Health in Challenging Times, Monograph 8. Sydney, Australia: NSW Transcultural Mental Health Centre.

Blackwell, D. (2005). Counselling and Psychotherapy with Refugees. London: Jessica Kingsley 
Bot, H. \& Wadensio , C. (2004). The Presence of a Third Party: A Dialogical View on Interpreter-Assisted Treatment. In B. Drozdek. \& J. P. Wilson (Eds), Broken Spirits ; The Treatment of Traumatized Asylum Seekers, Refugees, War and Torture Victims. New York: Brunner-Routledge.

Dearnley, B. (2000). Psycho-therapy in translation. One clinicians experience of working with interpreters. Society of Psychoanalytical Marital Psychotherapists 7 19-22.

Farooq, S. \& Fear, C. (2003). Working through Interpreters. Advances in Psychiatric Treatment, 9,104-109.

Haenal, F. (1997). Aspects and problems associated with the use of interpreters in psychotherapy of victims of torture. Torture 7 (3) 68-71.

Holder, R. (2002). The Impact of Mediated Communication on Psychological Therapy with Refugees and Asylum Seekers: Practitioners' Experiences. Unpublished dissertation: Department of Psychology, City University, London. Miller, K., Martell, Z., Pazdinek, L., Carruth, M. \& Lopez, F. (2005). The role of interpreters in psychotherapy with refugees: An exploratory study. American Journal of Orthopsychiatry, 75, 1, 27-39 
Patel, N. (2003). Speaking with the silent: Addressing issues of disempowerment when working with refugee people. In R. Tribe \& H. Raval, Working with Interpreters in Mental Health. Hove, East Sussex: BrunnerRoutledge

Raval, H. (1996). A systemic perspective on working with interpreters. Clinical Child Psychology and Psychiatry 1 (1) 29-43.

Rechtman, R. (2000). Stories of Trauma and Idioms of Distress: From Cultural Narratives to Clinical Assessment. Transcultural Psychiatry, 37,3, 403-415

Ruszczynski, S. (1993). The theory and practice of the Tavistock Institute of Marital Studies. In S. Ruszczynski (Ed), Psychotherapy with Couples, Theory and Practice at the Tavistock Institute of Marital Studies. London: Tavistock Clinic.

Sabin, J.E. (1975). Translating Despair. American Journal of Psychiatry, 132 (2) 197-199.

Saxthorp, V. \& Christiansen, J. (1991). Working with refugee families from the Middle East in Denmark. Presented at the III International Conference of Centres, Institutions and Individuals concerned with the Care of Victims of Organised Violence. Santiago: Chile 
Spector, S, Briedis, J. \& Rebori, V. The Triad of Interpreted Psychotherapy. Unpublished manuscript.

Tribe, R. (1997). A critical analysis of a support and clinical supervision group for interpreters working with refugees located in Britain. Groupwork 10 (3) $196-214$

Tribe, R. (1999) Bridging the gap or damming the flow? Bicultural Workers, Some observations on using interpreters when working with refugee clients, many of whom have been tortured. British Journal of Medical Psychology, $72,567-576$

Tribe, R. \& Thompson, K. (submitted). Opportunity for development or necessary nuisance? - The case for viewing working with interpreters as a bonus in therapeutic work.

Westermeyer, J. (1990). Working with an interpreter in psychiatric assessment and treatment. Journal of Nervous and Mental Disease, 178,12, 745- 749.

\footnotetext{
${ }^{\mathrm{i}}$ However, it is important for clinicians not to assume a cultural similarly between client and interpreter given the vast heterogeneity between individuals from the same national or ethnic background. Certainly, differences of gender, age, class, education, location (rural or urban) can make for radically different worldviews amongst groups sharing a common language
} 